\title{
Efficient narrowband terahertz generation in cryogenically cooled periodically poled lithium niobate
}

\author{
Sergio Carbajo, ${ }^{1-3}$ Jan Schulte, ${ }^{1,2}$ Xiaojun Wu, ${ }^{1,3}$ Koustuban Ravi, ${ }^{1,4}$ Damian N. \\ SCHIMPF, ${ }^{1,3}$ FRANZ X. KÄRTNER ${ }^{1-4}$ \\ ${ }^{1}$ Center for Free-Electron Laser Science, DESY, Notkestraße 85, Hamburg 22607, Germany \\ ${ }^{2}$ Department of Physics, University of Hamburg, Hamburg 22761, Germany \\ ${ }^{3}$ The Hamburg Center for Ultrafast Imaging, Luruper Chaussee 149, Hamburg 22761, Germany \\ ${ }^{4}$ Department of Electrical Engineering and Computer Science, and Research Laboratory of Electronics, Massachusetts Institute of Technology, \\ 77 Massachusetts Avenue, Cambridge, Massachusetts 02139, USA \\ Corresponding author: scarbajo@stanford.edu; franz.kaertner@desy.de
}

Received XX Month XXXX; revised XX Month, XXXX; accepted XX Month XXXX; posted XX Month XXXX (Doc. ID XXXXX); published XX Month XXXX

\begin{abstract}
We present an efficiency scaling study of optical rectification in cryogenically cooled periodically poled lithium niobate for the generation of narrowband terahertz radiation using ultrashort pulses. The results show an efficiency and brilliance increase compared to previous schemes of up to two orders of magnitude by exploring the optimal pump pulse format at around $800 \mathrm{~nm}$, and reveal saturation mechanisms limiting the conversion efficiency. We achieve $>10^{-3}$ energy conversion efficiencies, $\mu \mathrm{J}$-level energies, and bandwidths $<20 \mathrm{GHz}$ at $\sim 0.5 \mathrm{THz}$, thereby showing unprecedented spectral brightness in the 0.1-1 THz range relevant to terahertz science and technology.
\end{abstract}

OCIS codes: (190.7110) Ultrafast nonlinear optics; (140.3070)

Infrared and far-infrared lasers

http://dx.doi.org/

An increasingly wide variety of applications in fundamental sciences and advanced technologies makes use of coherent terahertz waves, mainly due to their ability to stimulate distinct structural dynamics and properties of matter, such as inter- and intra-molecular excitations [1, 2], ultrafast phase-changes [3], chirality switching [4], or molecular orientation and alignment [5], among others. Many of these applications demand the development of narrowband terahertz radiation sources because they can select, switch, or control processes in distinctly defined frequency bands. A well-established source for narrowband and tunable terahertz emission is the gyrotron, a device capable of delivering MW-level terahertz power in continuous-wave operation up to $\sim 200 \mathrm{GHz}$. When pulsed, gyrotrons typically emit pulses as short as nanoseconds at $\mathrm{Hz}$ repetition rates, thereby restricting their use to slow or static processes. In the pursuit of accessing future novel ultrafast applications, such as time-resolved microscopy [6] or electron acceleration [7], the promise of generating efficient high-field, narrowband, tunable and ultrafast terahertz radiation lies in laser-based techniques. Generation methods include optical parametric oscillation (OPO), difference frequency generation (DFG) with two distinct narrowband lasers, and optical rectification (OR) of a single broadband pulse (or intra-pulse DFG) in periodically poled (PP) crystals. Previous DFG work has proven successful through different schemes in achieving optical-to-terahertz energy conversion efficiencies in the order of $\sim 10^{-5}[8,9]$. OPOs have demonstrated slightly better conversion efficiencies [10], but cast doubt about their frequency and bandwidth tunability, with difficulty to reach bandwidths narrower than a few hundreds of $\mathrm{GHz}$ in the lower terahertz frequency regime [11]. Recent work in seeded parametric generation in bulk lithium niobate has shown remarkable progress on wide tuning range terahertz generation with efficiencies up to $10^{-4}$ [12].

Alternatively, OR in PP crystals was also demonstrated to produce frequency and bandwidth tunable terahertz radiation [13-16]. Here, a broadband optical pulse is rectified by a quasi-phase matched (QPM) material with alternating sign of second-order susceptibility $\chi^{(2)}$ [17], which enhances conversion efficiency by achieving longer interaction lengths in collinear geometries. In this process the down-converted central terahertz frequency $\left(v_{0}\right)$ is primarily determined by the domain period $(\Lambda)$ and the angle of observation $(\Theta)$-defined as the angle relative to the normal of the lateral surface-, and to a lesser extent by temperature $(T)$ tuning via temperature dependent index of refraction $(\partial n / \partial T)$. That is, the second-order polarizability causes terahertz emission with forward $(\Theta=\pi / 2)$, backward $(\Theta=-\pi / 2)$, and perpendicular $(\Theta=0)$ components relative to the pump k-vector at a frequency given by

$$
v_{0}=\frac{c}{\Lambda\left|n_{p}-n_{T H z} \sin \Theta\right|}
$$

where $c$ is the speed of light in vacuum, and $n_{p}$ and $n_{T H z}$ are the group index of the pump and refractive index of the terahertz wave, respectively. The frequency $v_{0}$ can be tuned via poling-period and/or temperature tuning. The shape of the generated terahertz waveform is influenced by the domain structure of the PP crystal. The relative spectral bandwidth $\left(\Delta v_{0} / v_{0}\right)$ decreases with increasing the number of domains $(N)$ [15]. Previous experimental demonstrations of this technique used femtosecond pump pulses in periodically-poled lithium niobate (PPLN) [13] and lithium tantalate [15]. At higher frequencies, where terahertz absorption is strong, surface 
emission was proposed in order to shorten the terahertz travel path inside the material while maintaining its longitudinal length [14].

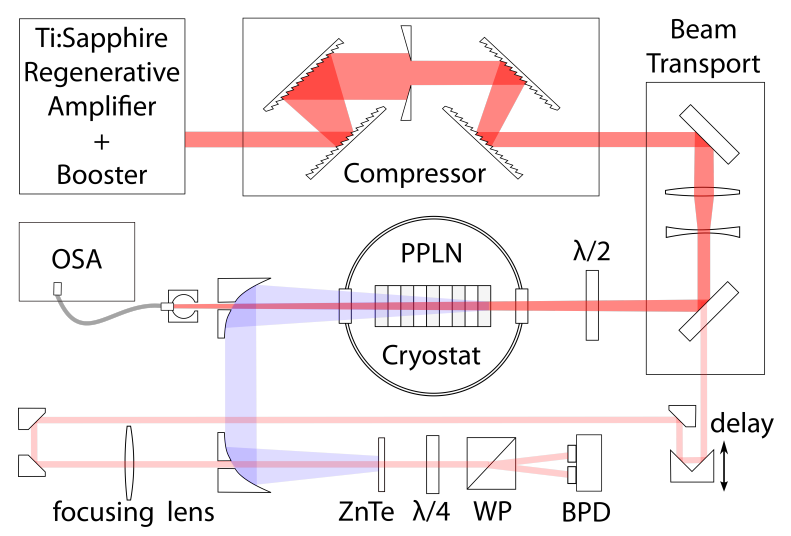

Fig. 1. Schematic of the experimental setup in EO-sampling configuration. OSA: optical spectrum analyzer; WP: Wollaston prism; BPD: balanced photodiode.

OR in PP crystals has the potential of producing high energy terahertz pulses with narrow bandwidth. In this letter, we demonstrate an efficiency increase of about two orders of magnitude compared with data from previous OR experiments in PP crystals reporting conversion efficiencies of $10^{-5}[13,15]$. Here, the optimization strategy consists of the following: (i) reducing the terahertz absorption of lithium niobate by cryogenic cooling; (ii) optimizing the pump pulse bandwidth; and (iii) exploring the impact of crystal length on efficiency saturation. To reveal design strategies, we conduct systematic measurements of the extracted terahertz power, energy, and efficiency, as well as the temporal waveform and corresponding spectrum as a function of five characteristic parameters, namely, average intensity $(I)$-defined as the energy inside the crystal divided by the temporal pulse duration (Gaussian FWHM) and beam mode area $\left(\pi w^{2}\right)-$, bandwidth $(\Delta \lambda)$, geometric crystal length $(L)$, domain period $(\Lambda)$, and temperature $(T)$.

As illustrated in Fig. 1, we employ compressed pulses from a Ti:Sapphire laser amplifier. The system outputs mJ-level pulses at $1 \mathrm{kHz}$ repetition rate at a wavelength around $800 \mathrm{~nm}$. A variable slit in the grating-pair compressor controls the bandwidth, ranging approximately between 3.5 and $10 \mathrm{~nm}$. Without the slit the laser delivers $150 \mathrm{fs}$ pulses. All QPM structures are made of 5mol.\%-doped congruent z-cut PPLN crystals. Each crystal is chromium-coated on all lateral surfaces and placed in a cryostat chamber pressure-connected to a $\mathrm{LN}_{2}$ dewar. The temperature is measured using a silicon diode sensor glued to the crystal surface. At the PPLN input facet, the mode profile of the pump is $2.7 \mathrm{~mm}$ in diameter at $1 / \mathrm{e}^{2}$. The terahertz radiation exits the cryostat chamber through a $3.5 \mathrm{~mm}$ thick polymethylpentene (TPX) window. The chamber pressure level is lower than $10^{-5}$ mbar. The extracted radiation is collected and transported through two 90-degree off-axis parabolas with 2" apertures and $\mathrm{NA}=1$ in a $4 \mathrm{f}$-configuration. At the terahertz image plane, the temporal waveform of the extracted terahertz spectrum is measured through electro-optic (EO) sampling in $0.5 \mathrm{~mm}$-thick ZnTe using a small fraction of the pump beam as the EO-probe. At the same image plane, albeit not depicted in Fig. 1, the extracted terahertz power is measured with both a pyroelectric detector (Gentec-EO Model THZ-B) and a calibrated thermal power sensor (Ophir-Spiricon Model 3A-P-THz). In the following, the reported efficiency is directly calculated from the measured power without compensating for Fresnel losses at the PPLN output facet $(\sim 45-48 \%)$ or transmission losses through the TPX outcoupling window ( 18-22\%). Note that a through-hole in the first collecting parabola serves as an outlet for the pump beam. The beam is then collected by an integrating sphere fiber-coupled to an optical spectrum analyzer to monitor the pump spectrum shaped by the inherent down-conversion of OR.

In the first set of measurements, we study the effect of crystal temperature. Cryogenic-cooling is known to reduce the terahertz linear absorption of lithium niobate [15]. This reduction is relevant in QPM structures since longer nonlinear interaction lengths may enhance conversion efficiency before absorption imposes limitations. As shown in Fig. 2.a, we measure a five-fold increase in extracted energy from the same PPLN crystal ( $\mathrm{L}=5 \mathrm{~mm}$ and $\Lambda=212 \mu \mathrm{m}$ ) when reducing its temperature from $295 \mathrm{~K}$ to $100 \mathrm{~K}$. In this data set, the PPLN is pumped with the full laser bandwidth of about $10 \mathrm{~nm}$. The peak emission frequency corresponding to the $295 \mathrm{~K}$ spectrum (Fig. 2.b) is $520 \mathrm{GHz}$, while at $100 \mathrm{~K}$ it shifts to $559.4 \mathrm{GHz}$ due to the change in refractive index with temperature. As shown in Fig. 2.b, the backward-propagating terahertz spectral components emerge around $200 \mathrm{GHz}$, most notably at lower temperatures because of the reduced absorption. It is easily deduced that back-propagating $200 \mathrm{GHz}$ radiation is phase-matched if one sets $\theta=-\pi / 2$ in Eq.(1). Fig. 2.c-d show the temporal waveforms retrieved by EO-sampling at $295 \mathrm{~K}$ and $100 \mathrm{~K}$, respectively. The normalized temporal waveforms are color-overlaid in the forward- (magenta) and backward-propagating (blue) components with their relative field strengths. Higher terahertz absorption at room temperature severely weakens the EO-sampled backward-propagating wave (Fig. 2.c. in blue). Conversely, at cryogenic temperatures (Fig. 2.d), the backward-propagating waves are again prominently visible at $\sim 58 \mathrm{ps}$, a delay with respect to the forward-propagating wave that corresponds to the reflection of the backward-propagating wave at the entrance facet of the PPLN and then propagating over one entire crystal length before it exits its output facet.

The bandwidth of the pump has a strong effect on the amount of energy transferred into the fundamental QPM terahertz wave. Previous analytical studies have already shown efficiency tradeoffs between pump fluence and bandwidth [17], resulting in efficiencies peaking at about $4 \mathrm{~nm}$ bandwidth in the current range of parameters. The optimal bandwidth may be understood as follows. For a given $=$ terahertz frequency (determined by $\Lambda$ ) the spectral bandwidth of the pump laser must support the right frequency spacing for down-conversion. For bandwidths smaller than the optimum, there are not enough photon-pairs at the right frequency spacing. For bandwidths larger than the optimum, dephasing effects take place as well as energy transfer into odd-harmonics of the fundamental terahertz wave due to high grating harmonics of the poling. We investigate the efficiency $(\eta)$ extracted from the same PPLN crystal $(\mathrm{L}=5 \mathrm{~mm}$; $\Lambda=212 \mu \mathrm{m} ; \mathrm{T}=100 \mathrm{~K}$ ) at around $500 \mathrm{GHz}$ as a function of the bandwidth of the $800 \mathrm{~nm}$ pump pulse. Fig. 3.a shows 
the energy efficiency curves corresponding to different pump bandwidth as a function of pump intensity. These results are depicted in Fig. 3.b for a fixed intensity of $50 \mathrm{GW} / \mathrm{cm}^{2}$. A maximum energy conversion efficiency of $0.12 \%$ is achieved at around $6.5 \mathrm{~nm}$. The increase in optical-to-terahertz energy transfer is also observed in the red-shifted pump spectrum (Fig. 3.c.). This few-nm red-shift arises from cascaded down-conversion of optical photons, which undergo more cascaded cycles as the process becomes more efficient.

a)

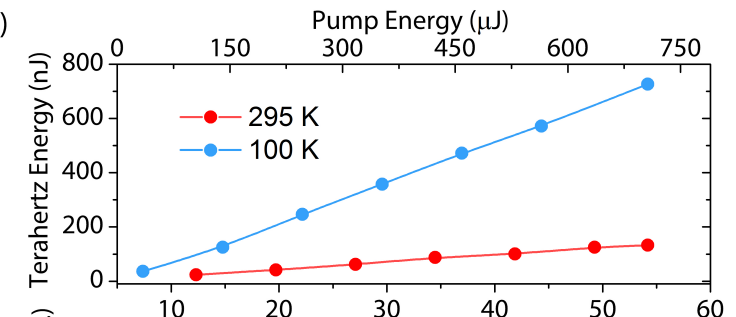

b)
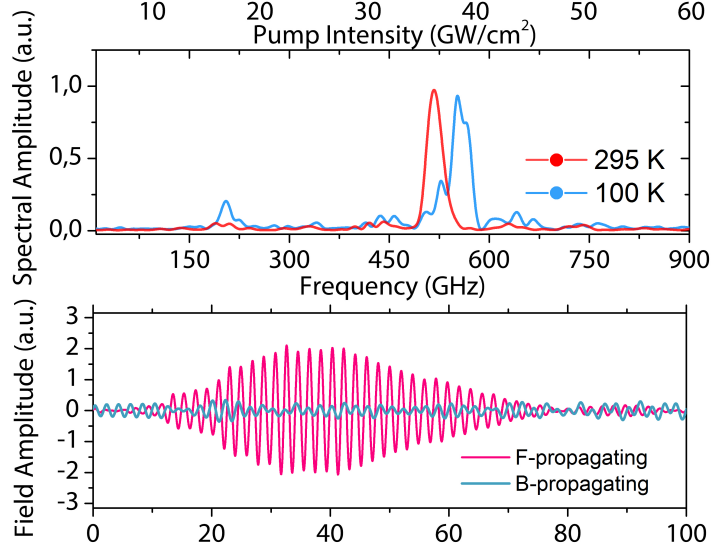

d)

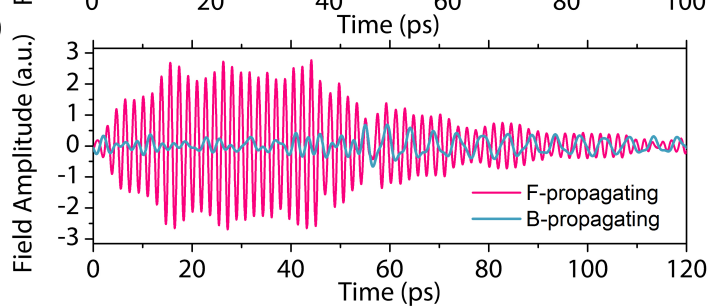

Fig. 2. Influence of temperature ( $\mathrm{L}=5 \mathrm{~mm} ; \Lambda=212 \mu \mathrm{m})$. a) Terahertz energy as a function of pump energy and intensity; b) corresponding normalized terahertz spectra with peak frequencies $v_{0}=520 \mathrm{GHz} \quad(\mathrm{T}=300 \mathrm{~K})$ and $\left.v_{0}=559.4 \mathrm{GHz}(\mathrm{T}=100 \mathrm{~K}) ; \mathrm{c}\right)$ and $\left.\mathrm{d}\right)$ forward $-(v>375 \mathrm{GHz})$ and backward-propagating $(v \leq 375 \mathrm{GHz})$ decomposed temporal waveforms at $295 \mathrm{~K}$ and $100 \mathrm{~K}$, respectively.

At cryogenic temperatures, the terahertz absorption coefficient at around $0.5 \mathrm{THz}$ is sufficiently small (as shown in Fig. 2) that the conversion efficiency benefits from longer crystal lengths $(L)$. Longer $L$ with same $\Lambda$ ( $L=N \Lambda$ ) would also yield emission with narrower linewidth, since $\Delta v \propto N^{-1}$. However, it is expected that the interplay between the pump intensity $I$ and $L$ may limit the down-conversion process. For instance, self-focusing and self-phase modulation may act upon the spatio-temporal phase of the pump pulse at sufficiently high intensities. The roles of these and other mechanisms need further investigation.
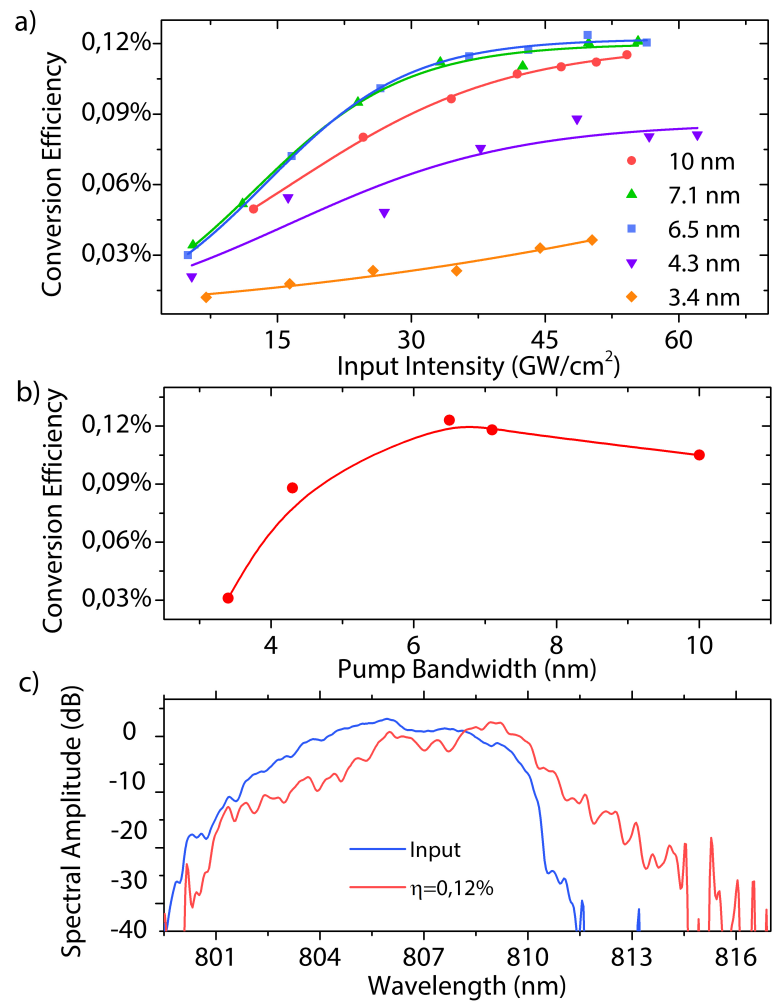

Fig. 3. Influence of pump pulse format $(\mathrm{L}=5 \mathrm{~mm}$; $\Lambda=212 \mu \mathrm{m} ; \mathrm{T}=100 \mathrm{~K}$ ). a) Extracted efficiency as a function of pump intensity for bandwidths ranging from 3.4 to $10 \mathrm{~nm}$. b) Efficiency as a function of bandwidth for a pump intensity of $50 \mathrm{GW} / \mathrm{cm}^{2}$. c) Recorded input and output optical spectra for highest achieved conversion efficiency (at $\Delta \lambda=6.5 \mathrm{~nm}$ and $\mathrm{I}=50 \mathrm{GW} / \mathrm{cm}^{2}$ ).

In order to investigate the effect of crystal length, we measure the terahertz energy and efficiency with respect to pump intensity for three different crystal lengths $(L=5,10,20 \mathrm{~mm})$ and same domain period $(\Lambda=212 \mu \mathrm{m})$ at 100K. As shown in Fig. 4.a, the terahertz energy increases monotonically with pump intensity for all three $L$ values. The $20 \mathrm{~mm}$ PPLN shows saturation of the conversion efficiency (Fig. 4.b) that is more pronounced compared with the two other PPLNs. The scaling trend of the efficiency curve is best for the $5 \mathrm{~mm}$ crystal, and begins to roll off at intensities of about $30 \mathrm{GW} / \mathrm{cm}^{2}$. Note that in this regime the photo-generated carrier concentration -which can severely absorb terahertz radiation- due to three photon absorption in lithium niobate is negligibly small [18]. Also, the pump power is two orders of magnitude above the critical power for self-focusing of about $10 \mathrm{MW}$,

Table 1. Summary of operational system specifications at cryogenic and room temperature

\begin{tabular}{|c|c|c|c|c|c|c|c|c|c|c|c|}
\hline \multicolumn{3}{|c|}{ QPM crystals } & \multicolumn{3}{|c|}{ Optical pump } & \multicolumn{6}{|c|}{ Terahertz waves } \\
\hline \multirow{2}{*}{$\Lambda[\mu \mathrm{m}]$} & \multirow{2}{*}{$\mathrm{L}[\mathrm{mm}]$} & \multirow{2}{*}{$\mathrm{N}$} & \multirow{2}{*}{$\Delta \lambda[\mathrm{nm}]$} & \multirow{2}{*}{$\mathrm{t}[\mathrm{fs}]^{+}$} & \multirow{2}{*}{$\mathrm{I}\left[\mathrm{GW} / \mathrm{cm}^{2}\right]$} & \multicolumn{2}{|c|}{$v_{0}[\mathrm{GHz}]$} & \multirow{2}{*}{$\frac{\Delta v}{295 \mathrm{~K}}$} & \multirow{2}{*}{$\begin{array}{c}{[\mathrm{GHz}]} \\
100 \mathrm{~K} \\
\end{array}$} & \multicolumn{2}{|c|}{$\eta\left[\times 10^{-3}\right]$} \\
\hline & & & & & & $295 \mathrm{~K}$ & $100 \mathrm{~K}$ & & & $295 \mathrm{~K}$ & $100 \mathrm{~K}$ \\
\hline 400 & 10 & 25 & \multirow{5}{*}{$6-7$} & \multirow{5}{*}{$190-160$} & \multirow{5}{*}{$50-60$} & 275.8 & - & 95.6 & - & 0.28 & - \\
\hline \multirow{3}{*}{212} & 5 & 24 & & & & 520.0 & 559.4 & 27.7 & 31.4 & 0.36 & 1.20 \\
\hline & 10 & 48 & & & & 517.5 & 561.8 & 19 & 18.6 & 0.19 & 1.03 \\
\hline & 20 & 94 & & & & - & 559.8 & - & 11.2 & 0.04 & 0.89 \\
\hline 125 & 10 & 80 & & & & 867.3 & - & 35 & - & 0.12 & - \\
\hline
\end{tabular}



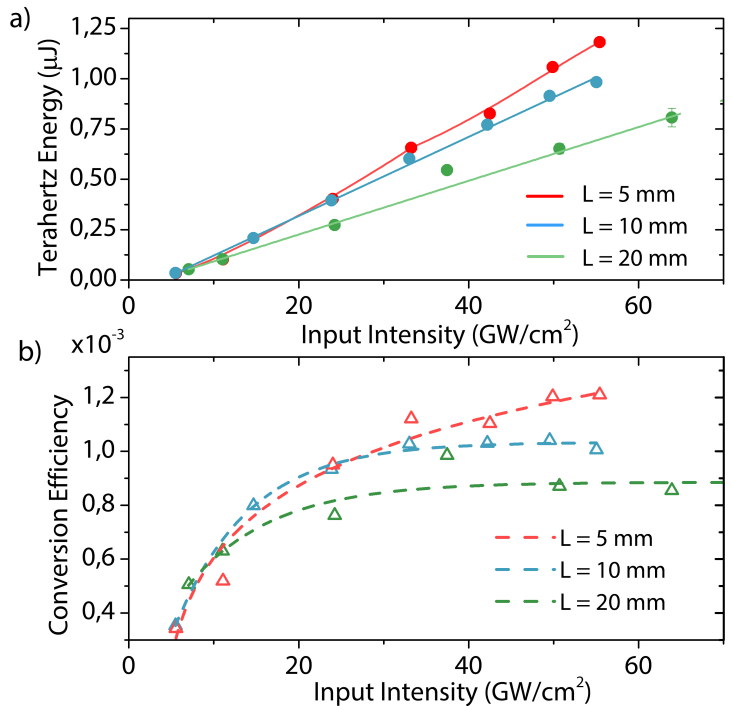

Fig. 4. Terahertz energy a) and conversion efficiency b) $(\mathrm{T}=100 \mathrm{~K} ; \quad \Lambda=212 \mu \mathrm{m} ; \Delta \lambda=6.5 \mathrm{~nm})$ for $\mathrm{L}=5$, 10 , and $20 \mathrm{~mm}$. QPM peak emission is $v_{0} \sim 520 \mathrm{GHz}$ for all three crystal lengths. Solid and dashed splines are visual interpretative guidelines.

and there should be significant self-phase modulation due to large B-integral values. These nonlinear effects deteriorate the quality of the pump towards the end of the crystal (not shown). This may limit efficiency scaling using longer crystals due to a deteriorated pump where terahertz waves propagate with non-negligible absorption. Such effects unfold in the measured temporal waveforms (Fig. 2). At room temperature, the trailing part of the waveform -which is generated first in the process- decays as expected due to linear absorption in the PPLN. Without spatio-temporal beam distortions, the leading part of the waveform ought to reach the highest field amplitude, since it is generated last. However, the measurements show that the field amplitude too decays at the leading edge at cryogenic temperatures (Fig. 2.d). The amplitude decay at the leading part of the waveform confirms that the down-conversion process becomes inefficient towards the end of crystal due to distortions of the pump beam.

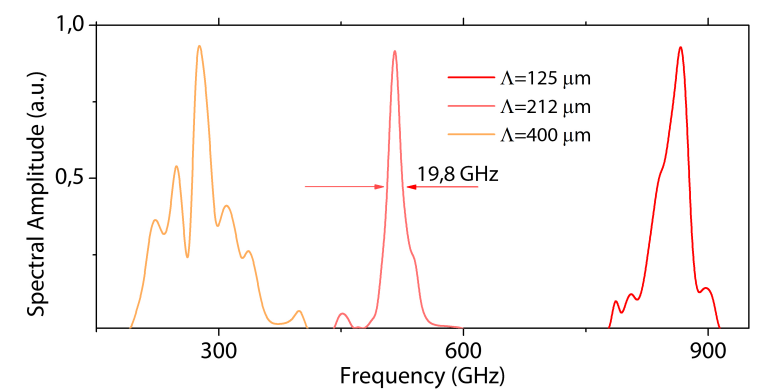

Fig. 5 - Spectral amplitude of $10 \mathrm{~mm}$ crystals ( $\mathrm{T}=295 \mathrm{~K}$ ). The peak frequencies are $v_{0}=276,513$, and $867 \mathrm{GHz}$, for the domain periods 400,212 , and $125 \mu \mathrm{m}$, respectively.

Exploiting the low absorption coefficient of lithium niobate at cryogenic temperatures and optimizing the pump bandwidth permits terahertz generation with $\eta$ of $10^{-4}$ to $10^{-3}, v_{0}$ in the $0.1-1 \mathrm{THz}$ range and $\Delta v$ from 10 to $100 \mathrm{GHz}$. Fig. 5 exemplifies this capability with spectra acquired employing $10 \mathrm{~mm}$ long PPLNs with different poling periods. Table 1 provides a more complete overview of measured parameters for different $L$ and $\Lambda$.
There, the terahertz linewidths are the FWHM values of Lorentzian line shape fits of the spectral amplitude.

In conclusion, we have scaled the conversion efficiency of narrowband terahertz generation in PPLN to above $10^{-3}$ in the sub-THz frequency range. These results were achieved by rectifying high peak-power ultrashort pulses. At frequencies around $0.5 \mathrm{THz}$, pumping of cryogenically cooled PPLN crystals with optimized spectral bandwidth (6-7 $\mathrm{nm}$ for $800 \mathrm{~nm}$ pulses) resulted in $0.12 \%$ efficiency and more than $1 \mu \mathrm{J}$ of terahertz pulse energy. Based on the current experiment and crystals used, it appears that extending the crystal length beyond $10 \mathrm{~mm}$ is disadvantageous for efficiency scaling due to limitations imposed by the combination of terahertz absorption and spatial-temporal distortions of the pump. Detailed causes of such distortions are subject of ongoing research.

Funding. This work was supported through the excellence cluster "The Hamburg Centre for Ultrafast ImagingStructure, Dynamics and Control of Matter at the Atomic Scale" of the Deutsche Forschungsgemeinschaft and through the European Research Council under the European Union's Seventh Framework Programme (FP/2007-2013)/ERC Synergy Grant Agreement no. 609920. We would like to thank Oliver Mücke, Giovanni Cirmi, and Giulio Rossi for their support with the laser source.

[1] K.N. Egodapitiya, S. Li, R.R. Jones, Physical Review Letters, 112 (2014) 103002.

[2] M. Jewariya, M. Nagai, K. Tanaka, Physical Review Letters, 105 (2010) 203003.

[3] M. Liu, H.Y. Hwang, H. Tao, A.C. Strikwerda, K. Fan, G.R. Keiser, A.J. Sternbach, K.G. West, S. Kittiwatanakul, J. Lu, S.A. Wolf, F.G. Omenetto, X. Zhang, K.A. Nelson, R.D. Averitt, Nature, 487 (2012) 345-348.

[4] S. Zhang, J. Zhou, Y.-S. Park, J. Rho, R. Singh, S. Nam, A.K.

Azad, H.-T. Chen, X. Yin, A.J. Taylor, X. Zhang, Nat Commun, 3 (2012) 942.

[5] S. Fleischer, Y. Zhou, R.W. Field, K.A. Nelson, Physical Review Letters, 107 (2011) 163603.

[6] T.L. Cocker, V. Jelic, M. Gupta, S.J. Molesky, A.J.

BurgessJacob, G.D.L. Reyes, L.V. Titova, Y.Y. Tsui, M.R. Freeman, F.A. Hegmann, Nat Photon, 7 (2013) 620-625. [7] L.J. Wong, A. Fallahi, F.X. Kärtner, Opt. Express, 21 (2013) 9792-9806.

[8] W. Shi, Y.J. Ding, Applied Physics Letters, 83 (2003) 848-850.

[9] K. Miyamoto, H. Minamide, M. Fujiwara, H. Hashimoto, H. Ito, Opt. Lett., 33 (2008) 252-254.

[10] T. Taniuchi, S. Okada, H. Nakanishi, Journal of Applied Physics, 95 (2004) 5984-5988.

[11] J.E. Schaar, K.L. Vodopyanov, M.M. Fejer, Opt. Lett., 32 (2007) 1284-1286.

[12] S.i. Hayashi, K. Nawata, T. Taira, J.-i. Shikata, K. Kawase, H. Minamide, Sci. Rep., 4 (2014).

[13] Y.-S. Lee, T. Meade, V. Perlin, H. Winful, T.B. Norris, A. Galvanauskas, Applied Physics Letters, 76 (2000) 2505-2507.

[14] C. Weiss, G. Torosyan, Y. Avetisyan, R. Beigang, Opt. Lett., 26 (2001) 563-565.

[15] N.E. Yu, K.S. Lee, D.K. Ko, C. Kang, S. Takekawa, K. Kitamura, Optics Communications, 284 (2011) 1395-1400.

[16] K. L. Vodopyanov, X. Yu, J. S. Harris, Y.-S. Lee, W. C. Huribut, V. G. Kozlov, D. Bliss, C. Lynch, Applied Physics Letters, 89 (2006) 141119.

[17] K.L. Vodopyanov, Opt. Express, 14 (2006) 2263-2276.

[18] B.S. Wherrett, J. Opt. Soc. Am. B, 1 (1984) 67-72. 
1. Egodapitiya, K.N., S. Li, and R.R. Jones, Terahertz-Induced Field-Free Orientation of Rotationally Excited Molecules. Physical Review Letters, 2014. 112(10): p. 103002.

2. Jewariya, M., M. Nagai, and K. Tanaka, Ladder Climbing on the Anharmonic Intermolecular Potential in an Amino Acid Microcrystal via an Intense Monocycle Terahertz Pulse. Physical Review Letters, 2010. 105(20): p. 203003.

3. Liu, M., et al., Terahertz-field-induced insulator-to-metal transition in vanadium dioxide metamaterial. Nature, 2012. 487(7407): p. 345-348.

4. Zhang, S., et al., Photoinduced handedness switching in terahertz chiral metamolecules. Nat Commun, 2012. 3: p. 942.

5. Fleischer, S., et al., Molecular Orientation and Alignment by Intense Single-Cycle $\mathrm{THz}$ Pulses. Physical Review Letters, 2011. 107(16): p. 163603.

6. Cocker, T.L., et al., An ultrafast terahertz scanning tunnelling microscope. Nat Photon, 2013. 7(8): p. 620-625.

7. Wong, L.J., A. Fallahi, and F.X. Kärtner, Compact electron acceleration and bunch compression in THz waveguides. Optics Express, 2013. 21(8): p. 9792-9806.

8. Shi, W. and Y.J. Ding, Continuously tunable and coherent terahertz radiation by means of phase-matched difference-frequency generation in zinc germanium phosphide. Applied Physics Letters, 2003. 83(5): p. 848-850.

9. Miyamoto, K., et al., Widely tunable terahertz-wave generation using an $\mathrm{N}$-benzyl-2-methyl-4-nitroaniline crystal. Optics Letters, 2008. 33(3): p. 252-254.

10. Taniuchi, T., S. Okada, and H. Nakanishi, Widely tunable terahertz-wave generation in an organic crystal and its spectroscopic application. Journal of Applied Physics, 2004. 95(11): p. 5984-5988.

11. Schaar, J.E., K.L. Vodopyanov, and M.M. Fejer, Intracavity terahertz-wave generation in a synchronously pumped optical parametric oscillator using quasi-phase-matched GaAs. Optics Letters, 2007. 32(10): p. 1284-1286.

12. Hayashi, S.i., et al., Ultrabright continuously tunable terahertz-wave generation at room temperature. Sci. Rep., 2014. 4.

13. Lee, Y.-S., et al., Generation of narrow-band terahertz radiation via optical rectification of femtosecond pulses in periodically poled lithium niobate. Applied Physics Letters, 2000. 76(18): p. 2505-2507.

14. Weiss, C., et al., Generation of tunable narrow-band surface-emitted terahertz radiation in periodically poled lithium niobate. Optics Letters, 2001. 26(8): p. 563-565.

15. Yu, N.E., et al., Temperature dependent narrow-band terahertz pulse generation in periodically poled crystals via difference frequency generation. Optics Communications, 2011. 284(5): p. 1395-1400.

16. K. L. Vodopyanov, M.M.F., X. Yu, J. S. Harris, Y.-S. Lee, W. C. Huribut, V. G. Kozlov, D. Bliss, C. Lynch, Terahertz-wave generation in quasi-phase matched GaAs. Applied Physics Letters, 2006. 89: p. 141119.

17. Vodopyanov, K.L., Optical generation of narrow-band terahertz packets in periodically inverted electro-optic crystals: conversion efficiency and optimal laser pulse format. Optics Express, 2006. 14(6): p. 2263-2276.

18. Wherrett, B.S., Scaling rules for multiphoton interband absorption in semiconductors. Journal of the Optical Society of America B, 1984. 1(1): p. 67-72. 